\title{
A Non-cooperative Game Theoretic Approach to Energy-efficient Power Control in Wireless Sensor Networks
}

\author{
Guoyan Yang and Xin Guan \\ College of Information Science and Technology, Heilongjiang University \\ Harbin P. R. China, 150080 \\ hdyangguoyan@gmail.com
}

\begin{abstract}
Aiming at the limited energy characteristics of wireless sensor networks, we apply game theory to solve the power control problem to reduce energy consumption in wireless sensor networks. In this paper, a distributed power control algorithm based on non-cooperative game theory under incomplete information is proposed, which adopts Signal-to-Interference Noise Ratio (SINR) as utility function. The purpose of power control algorithm for noncooperative game is to achieve the largest utility by optimal power control strategy, thus improve the total network energy efficiency. Moreover, Bayesian Nash equilibrium theorem is introduced to study the existence and uniqueness proof of Nash equilibrium algorithm. Simulation results show that there exist points for each of the cost functions considered, which give the maximum net utility given the strategies taken by all other nodes as fixed. And the proposed algorithm is efficient and can achieve better performance.
\end{abstract}

Keywords: Power control, non-cooperative game theory, utility function, Wireless sensor network

\section{Introduction}

A wireless sensor network (WSN) is a group of specialized sensor nodes each of which is small, lightweight and portable with a communication infrastructure intended to monitor and record conditions at diverse locations. Generally sensors are battery powered and have feeble data processing capability and short radio range [1]. The resource-constraint temperament of WSNs in terms of their size, cost, weight and lifetime [2] is a key area of apprehension for most applications using WSN. Coupled with the inherent unreliability of the wireless channel, possible hostile environment in certain application-specific deployment regions and device unreliability of individual nodes, WSNs are subject to unique challenges for efficient power management to prolong network lifetime in addition to fulfilling sensing objectives of the application.

Energy efficiency and achieving reliability is a key issue in wireless sensor networks. Battery capacity is limited and it is usually impossible to replace them. Any operation performed on a sensor consumes energy, involving discharge of battery power. Hence battery power efficiency is a critical factor while considering the energy efficiency of WSN. The three domains of energy consumption in a sensor are sensing, data processing and data communication, out of which communication is the main consumer of energy. Hence transmission at optimal power level is very essential. Optimal transmit power level implies the power level which reduces the interference, increases the successful packet transmission and provides the desired quality of service. Maintaining the transmission power under control is furthermore favorable to decrease the packet collision probability, which if not leads to 
more retransmitted packets wasting even more energy. Hitherto energy efficiency has been investigated extensively and various approaches to achieve an energy efficient network includes, scheduling sensor nodes to alternate between energy-conserving modes of operation, competent routing algorithms, clustering, incorporating astuteness and use of spatial localization at every node to lessen transmission of redundant data. Nodes specialization to different roles such as idle, sensing, routing and routing/sensing to maximize the utility of the nodes [3] has been proposed. An approach for optimizing transmit power for an ad-hoc network scenario [4] where all the nodes uses a uniform transmit power, and numerical results of transmit power sufficient to satisfy the network connectivity has been proposed. In recent years there has been a growing interest in applying game theory to study wireless systems [5], which used game theory to investigate power control and rate control for wireless data. The authors provide motivations for using game theory to study communication systems, and in particular power control [6]. Distributed iterative power control algorithms have been proposed for cellular networks; these algorithms examine to find the power vector for all the nodes that minimizes the total power with good convergence. Decentralized, game theoretic adaptive mechanisms, which can be deployed to manage sensor activities with low coordination overhead has been explained [7].

Game theory is a theory of decision making under conditions of uncertainty and interdependence. We now provide a brief introduction of the basics of game theory with the help of examples modeled on scenarios prevalent in WSNs. Game theory offers models for distributed allocation of resources and thus provides a way of exploring characteristics of wireless sensor networks. Energy harvesting technologies essential for independent sensor networks using a non cooperative game theoretic technique [8] is analyzed. Nash equilibrium was projected as the solution of this game to attain the optimal probabilities of sleep and wake up states that were used for energy conservation. The energy efficiency problem in wireless sensor networks as the maximum network lifetime routing problem is looked upon [9]. Here the transmit power levels is adjusted to just reach the anticipated next hop receiver such that the energy consumption rate per unit information transmission can be reduced.

\section{Related Work}

The power control in a wireless sensor network deals with the selection of proper transmission power for each packet at each link in a distributed fashion. This is a complex and intriguing problem since the selection of the power level fundamentally affects many aspects of the operation of the network and its resulting performance, for instance the quality of the signal received at the receiver, the interference it creates for the other receivers and energy consumption at each node. In traditional emergency or military situations, the nodes in wireless sensor networks usually belong to the same authority with certain common objectives. To maximize the overall system performance, nodes usually work in a full cooperative way and can unconditionally follow the designated algorithm with a system optimization method [10-12].Recently, emerging applications of wireless sensor networks are envisioned in civilian and commercial usage, where nodes typically do not belong to a single authority and may not pursue a common cooperate is unrealistic for autonomous users, especially when there are conflicting interests among the user [13, 14]. The autonomous nature of this evidently affects many aspects in the design of wireless sensor networks including the section power level, which is the focus of study in this paper.

The current power control algorithm includes two mainly types, they are centralized power control and distributed power control. Comparing with the centralized power control, the 
distributed power control does not require a large-scale data management, uses the present carrier interference radio measurement and transmission power, and so needs very little information. Therefore the research on the distributed power control algorithm is particularly important. Distributed power control issue in multi-user environment can be described that, chooses transmitting power levels of $n$ users in the premise of not violating the interference temperature and in limited spectrum holes, so that makes the joint data transmission rate maximal [15].

In recent years, power control has received intense attention for ad-hoc wireless networks and wireless sensor networks. In [16], a power control method is described as a Markov chain. An interesting characterization of power control algorithms from a control theoretic perspective can be found in [17]. With reference to WSNs, the authors of [18] investigate power control in a cooperative diversity scheme, which includes sleeping disciplines for the nodes. Compared to these contributions, and references therein, our original approach consists in modeling the physical layer characteristics that are necessary to implement power control algorithms for off-the-shelf WSNs. the paper [19] proposes a power control scheme for the case in which there is only one primary user and several secondary users, the study [20], assuming that there is only one primary and one secondary user in the network, proposes an opportunistic transmission scheme that is based on the assumption that the cognitive user has knowledge of the channel coefficients for all links, and exploits this knowledge to tune her transmit power. The paper [21] considers a Poisson random network model, wherein each primary user has a reassigned probability to transmit in each available time slot, and develops a power control algorithm for the secondary users exploiting instantaneous and local spectrum opportunities without causing unacceptable interference to primary users; in the study [22], finally, the tool of geometric programming is used to develop a distributed power control algorithm aiming at maximization of the total capacity of the secondary network subject to an interference constraint to primary users.

Our work, using a non-cooperative game theory to solve power control problem in the high-load wireless sensor networks, proposes an incomplete information power control algorithm. We use each node's signal to noise ratio (SINR) as the utility function and we study the existence and uniqueness of Nash equilibrium. Simulation results show that he proposed algorithm is efficient and can achieve better performance.

\section{System Model}

In this paper, we apply non-cooperative game theory with incomplete information to solve the problem of power control in wireless sensor networks. To formulate the non-cooperative power control analytically, we first model the self-interest property for power control in wireless sensor networks. Nodes transmitting are the players. Non-cooperative game theory means how players select strategy in mutual influence to maximize benefits. Node uses the minimum power to achieve the highest signal to noise ratio. Node power selection depends on the power selection strategy of other nodes. However, in a distributed sensor network, none node knows the power selection strategy of other nodes.

Therefore, this paper uses incomplete information dynamic game power control model. Because each node can not freely increase their own power in order to achieve a successful transfer, it will not only increase the interference to other nodes, but also lead to its neighbor nodes increase their power for interference transmission. In order to control the behavior of non-cooperation, this paper designs a balanced game strategy. Assuming all nodes in the games are "rational", in non-cooperative game strategy, all nodes will choose the best strategy 
for power transmission. The purpose of power control algorithm for non-cooperative game is to achieve the largest utility by lowest power cost.

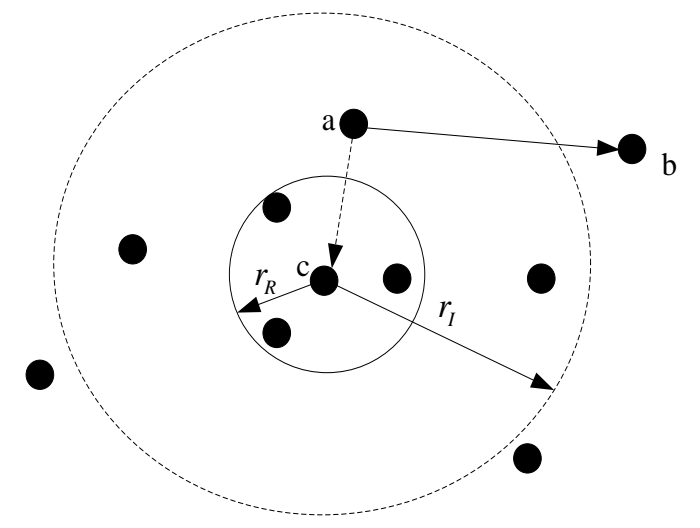

Figure 1. Interference at Node $c$ from a Local Neighbor Node a

Figure 1 shows that $c$ as the receiver under consideration. Node $a$, while transmitting to node $b$, acts as an interferer to node c. the reverse need not necessarily be true since the transmission power of node $a$ and nod $c$ can be different. We proceed to obtain an expression for the average number of potential interfering neighbors around a receiver. A rigorous treatment for the distribution of interference power can be found.

The non-cooperative game theory model can be described in three elements.

(1)Participants set $\Gamma=\{1,2,3 \ldots, \mathrm{n}\}$, which are $n$ nodes transmitting in the network.

(2) $\mathrm{s}=\left\{\mathrm{s}_{1}, \mathrm{~s}_{2}, \mathrm{~s}_{3}, \ldots, \mathrm{s}_{\mathrm{n}}\right\}, s_{i}$ is the strategy space for $i$ th node (i.e. any node $n_{i}$ can select the set of power values).

(3) Strategy profile $\mathrm{S}=\left\{\mathrm{S}_{1}, \mathrm{~S}_{2}, \mathrm{~S}_{3}, \ldots, \mathrm{S}_{\mathrm{N}}\right\}$, which are the strategies selected by all participants in the network according to their own utility function to maximize their benefits.

Any node $n_{i}$ 's utility function in the paper can be described as below. $s_{-i}$ are the strategy sets of all nodes except for the $n_{i}$.

$$
U_{i}\left(s_{i}, s_{-i}\right)=\frac{L R}{M s_{i}} f\left(\gamma_{i}\right) \frac{\text { bits }}{\text { joule }}
$$

$\gamma_{i}$ is the Signal-to-Interference Noise Ratio (SINR) defined as follows:

$$
\gamma_{i}=\frac{W}{R} \frac{h_{i} s_{i}}{\sum h_{i} s_{i}+\sigma^{2}}
$$

$W$ is available spread spectrum bandwidth, $R$ is transmission rate after spectrum spread. $\left\{h_{i}\right\}$ is path gain, $\sigma^{2}$ is variance of background noise. Assume which under certain path condition. $s_{i}$ is the transfer power of $n_{i}$. Define net utility function as follows: 


$$
U_{n e t}=\left\{\begin{array}{cc}
U_{i}\left(s_{i}, s_{-i}\right)-C\left(s_{i}\right) & \text { transmitting } \\
0 & \text { not transmitting }
\end{array}\right.
$$

Nodes access a wireless sensor networks through the air interface which is a common resource and they transmit information expending battery energy. Since the air interface is a shared medium, each node's transmission is a source of interference for others. The Signal-toInterference Noise Ratio (SINR) is a measure of the quality of signal reception for the wireless nodes. Typically, a node would like to achieve a high quality of reception (high SINR) while is at the same time expending a small amount of energy. Thus, it is possible to view both SINR and battery energy as commodities that a wireless node desires. There exists a tradeoff relationship between obtaining high SINR and low energy consumption. Finding a good balance between the two conflicting objectives is the primary focus of the power control component of radio resource management. This tradeoff is illustrated through the conceptual plot in Figure2.If the transmit power is fixed (fixed battery drain), with increasing the SINR of the node, the net utility increases. For sufficiently large SINR values, the error rate approaches zero which results in an asymptotic increase in net utility in the high SINR region. If the SINR is to be fixed, increasing the transmit power expedites the battery drain, which effectively reduces the satisfaction of the node.
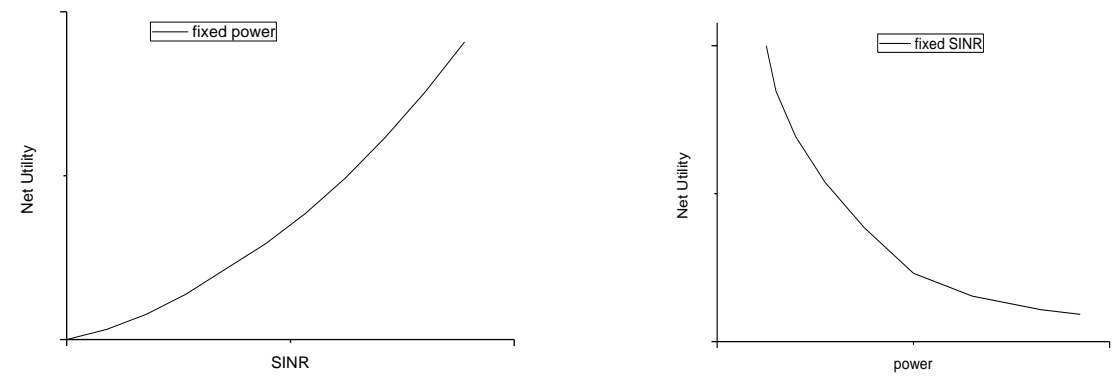

Figure 2. Net utility as a Function of SINR for Fixed Power and as a Function of Power for Fixed SINR

\section{Non-cooperative Power Control Game}

Because transmission power choice of transmission in sensor networks is the problem of non-cooperative game theory with incomplete information, we can have following result by using Bayesian Nash Equilibrium Method.

Theorem 1: Nash equilibrium exists and is unique in non-cooperative game with incomplete information of transmission power control.

Proof: Let $f_{s_{i}}(x)$ as the probability density function of $s_{i}$, assuming that node can carry out data transfer under any large power condition, that is when $s_{i} \rightarrow+\infty$, node transmission probability is 1 , so we can have $\int_{0}^{\infty} f_{s_{i}}(x) d x=1$.

But power game in the real world, in order to reduce the payments as well as reduce power costs, does not allow the node forward at any big power value. So we should let transmission power within a certain range, we can assume that when transmission power as $s_{i} \in\left[0, P_{i}\right]$, the 
$n_{i}$ will get the largest net utility. $P_{t}$ is the maximum power when a node transmits. So a node transmits at power $s_{i}$ and $s_{i} \in\left[0, P_{i}\right]$, then the probability that the nodes can transmit can be given by

$$
p\left(P_{t}\right)=\int_{0}^{P_{t}} f_{s_{i}}(x) d x
$$

The probability of no transmission is $1-p\left(P_{t}\right)$.So the probability that any $m$ nodes out of $N$ nodes are active is give by

$$
p_{m}=\left(\begin{array}{l}
N \\
m
\end{array}\right) p\left(P_{t}\right)^{m}\left(1-p\left(P_{t}\right)\right)^{N-m}
$$

Then the expected net utility of the $i$ th node transmitting is given by

$$
E\left[U_{i}^{n e t}\right]=\sum_{m=0}^{N}\left(u_{i}\left(s_{i}, s_{-i}\right)-A\left(s_{i}\right)\right) p_{m}
$$

Let $U_{i}\left(P_{t}\right)=\sum_{m=0}^{N} u_{i}(s i, s-i) p_{m}$, the expected net utility of $i$ th is given by

$$
E\left[U_{i}^{n e t}\right]=U_{i}\left(P_{t}\right)-C\left(s_{i}\right)
$$

If the nodes are transmitting, expected net utility is equation (8).If the node does not transmit the expected net utility is 0 . The expected net utility of any node is given by

$$
\begin{aligned}
G_{i}\left(P_{t}\right) & =\int_{z_{i}}^{P_{t}}\left[U_{i}\left(P_{t}\right)-C(x)\right] f_{s_{i}}(x) d x \\
& =U_{i}\left(P_{t}\right) p\left(P_{t}\right) \int_{z_{i}}^{P_{t}} C(x) f_{s_{i}}(x) d x
\end{aligned}
$$

Let $B\left(P_{t}\right)=\int_{z_{i}}^{P_{t}} C(x) f_{s_{i}}(x) d x$, then equation (8) can be written as

$$
G_{i}\left(P_{t}\right)=U_{i}\left(P_{t}\right) p\left(P_{t}\right)-B\left(P_{t}\right)
$$

From the equation (9) we can see that when the actual transmission power reach the value of upper bound, we get the same expected utility, i.e., $s_{i}=P_{t}$. Thus $P_{t}$ is the power upper bound of nodes transmitting when the whole network can achieve maximum utility. That is, $P_{t}$ is the solution to following equation

$$
U_{i}\left(P_{t}\right)-C\left(P_{t}\right)=0
$$

Theorem 2: Nash equilibrium exists is unique in non-cooperative game with incomplete information of transmission power control.

Proof: Let us suppose $T_{1}, T_{2}$ be transmission power of any node, of which $T_{1}$ is the solution to equation(10), $T_{2}$ is any power, and $T_{1} \neq T_{2}$, then the average utility of the node when $s_{i}=T_{1}$ and $s_{i}=T_{2}$ as follows, 
We can get the following equation

$$
\begin{aligned}
G_{i}\left(T_{1}\right) & =\int_{z_{i}}^{T_{1}}\left[U_{i}\left(T_{1}\right)-C(x)\right] f_{s_{i}}(x) d x \\
& =U_{i}\left(T_{1}\right) p\left(T_{1}\right)-B\left(T_{1}\right) \\
G_{i}\left(T_{2}\right) & =\int_{z_{i}}^{T_{2}}\left[U_{i}\left(T_{1}\right)-C(x)\right] f_{s_{i}}(x) d x \\
& =U_{i}\left(T_{1}\right) p\left(T_{2}\right)-B\left(T_{2}\right)
\end{aligned}
$$

$$
\begin{aligned}
G_{i}\left(T_{1}\right)-G_{i}\left(T_{2}\right) & =\left[C\left(T_{1}\right) p\left(T_{1}\right)-B\left(T_{1}\right)\right]-\left[C\left(T_{1}\right) \mathrm{p}\left(T_{2}\right)-B\left(T_{2}\right)\right] \\
& =C\left(T_{1}\right)\left[p\left(T_{1}\right)-p\left(T_{2}\right)\right]-\left[B\left(T_{1}\right)-B\left(T_{2}\right)\right]
\end{aligned}
$$

(1) when $T_{1}>T_{2}$, equation (11) can be written as

$$
\begin{aligned}
G_{\mathrm{i}}\left(T_{1}\right)-G_{\mathrm{i}}\left(\mathrm{T}_{2}\right) & =\int_{z_{i}}^{T_{1}}\left[U_{i}\left(T_{1}\right)-C(x)\right] f_{s_{i}}(x) d x-\int_{z_{i}}^{T_{2}}\left[U_{i}\left(T_{2}\right)-C(x)\right] f_{s_{i}}(x) d x \\
& =\int_{T_{2}}^{T_{1}}\left[C\left(T_{1}\right)-C(x)\right] f_{s_{i}}(x) d x
\end{aligned}
$$

Because $C\left(s_{i}\right)$ is monotone increasing function of power $s_{i}$, when $\forall x<T_{1}$, we get $C(x)<C\left(T_{1}\right)$. Therefore

$$
G i\left(T_{1}\right)-G i\left(T_{2}\right)>0
$$

(2) when $T_{1}<T_{2}$, equation (11) can be written as

$$
\begin{aligned}
G_{\mathrm{i}}\left(T_{1}\right)-G_{\mathrm{i}}\left(T_{2}\right) & =\int_{z_{i}}^{T_{1}}\left[U_{i}\left(T_{1}\right)-C(x)\right] f_{s_{i}}(x) d x-\int_{z_{i}}^{T 2}\left[U_{i}\left(T_{2}\right)-C(x)\right] f_{s_{i}}(x) d x \\
& =-\int_{T_{1}}^{T_{2}}\left[C\left(T_{1}\right)-C(x)\right] f_{i}(x) d x
\end{aligned}
$$

then $T_{1}<x \leq T_{2}$, we get $C\left(\mathrm{~T}_{1}\right)<C(x)$. Therefore $G_{\mathrm{i}}\left(T_{1}\right)-G_{\mathrm{i}}\left(T_{2}\right)>0$

Based on the above two cases, we can see that for any power $T_{2}$, if $\forall T_{2} \neq T_{1}$, then $G_{i}\left(T_{1}\right)>G_{i}\left(T_{2}\right)$, so $T_{1}$ is the selected power when the network achieves the maximum net utility, no power except for $T_{1}$ can provide expected utility. That is $T_{1}$ is the solution of incomplete information power control of Nash equilibrium as well as the unique solution.

\section{Numerical Results}

In order to evaluate the power control algorithm performance, we consider the cost component $C\left(s_{i}\right)$ that results due to transmitting at power $s_{i}$. To get the cost, we must consider $C\left(s_{i}\right)$ as the function of $s_{i}$.we consider two types of function which are linear and quadratic. That is $C\left(s_{i}\right)=k \times s_{i}$ (for linear) or $C\left(s_{i}\right)=k \times s_{i}{ }^{2}$ (for quadratic), where $k$ is a scaling factor. We show $C\left(s_{i}\right)$ in Figure2. 


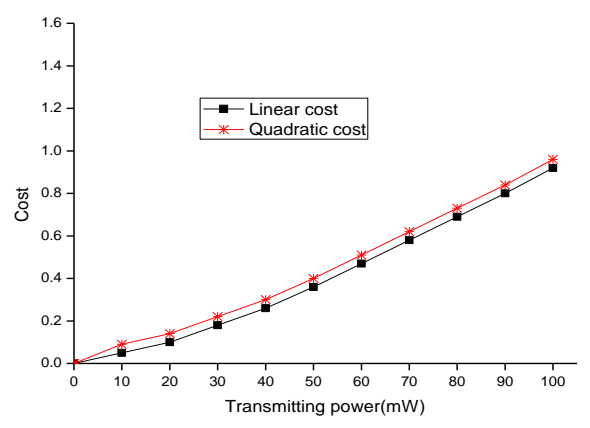

Figure 2. Different Cost Functions

In Figure3, we can observe the variation of the net utility with increasing transmitting power. It is intuitive that there will be an optimal value of $s_{i}$, beyond which the net utility will only decrease. This figure serves as a guideline for calculating the desired transmitting power to maximize net utility for a node $i$ transmitting to node $j$, given the strategies taken by all other nodes. For finding the best response to the strategies adopted by other nodes, we assume a subset of nodes to be active that are operating with fixed strategies.

We varied transmitting power from 1 to $100 \mathrm{~mW}$ in our calculation. This desired transmitting power level gives the best response for the node. If a node unilaterally changes its strategy and does not transmit with this transmitting power level, then the node will not get its best response and will not be able to reach Nash equilibrium even if a Nash equilibrium exists for this model.

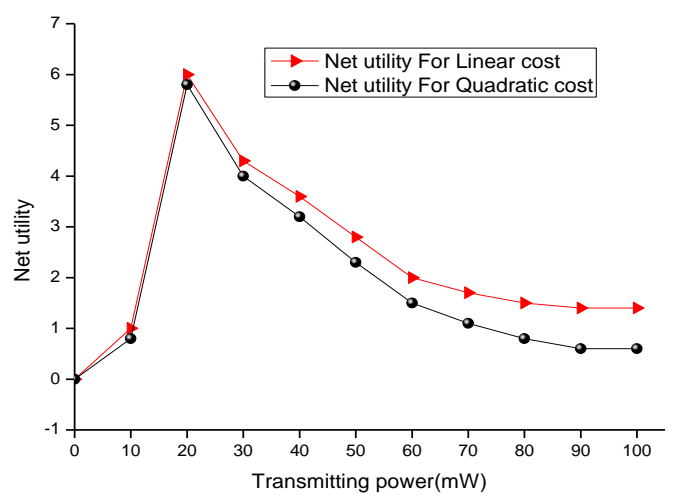

\section{Figure 3. Net Utility}

Energy efficiency of three algorithms are compared to evaluate the power control algorithm performance: (1) MAXPTC: maximum energy power control algorithm, in which all of the nodes use the maximum transmit power to send packets; (2) MINPTC: minimum energy power control Algorithm, in which all of the nodes use the minimum transmitting power to send packets; (3) The proposed algorithm: the non-cooperative power control algorithm in this paper. We mainly consider two performance parameters for energy efficiency and data transmission success rate. Energy efficiency: the total number of corrected received packets is divided by the total consumed energy during per unit time. Data 
transmission success rate: the total number corrected received packets are divided by the total number of transmitted packets.

The system is saturated. Simulation parameter settings are as follows:

Table 1. Simulation Parameter Settings

\begin{tabular}{|c|c|}
\hline Parameter statement & value \\
\hline Scenario size $/ \mathrm{m}^{2}$ & $100 \times 100$ \\
\hline Numbers of nodes & 30 \\
\hline Channel bandwidth $(W)$ & $1 \mathrm{MHZ}$ \\
\hline Channel rate $(R)$ & $20 \mathrm{kbps}$ \\
\hline Path gain $\left(h_{i}\right)$ & $7.75 \times 10^{-3} / \mathrm{d}_{\mathrm{i}}^{3.6}$ \\
\hline Background noise $\left(\sigma^{2}\right)$ & $5 \times 10^{-15} \mathrm{~W}$ \\
\hline
\end{tabular}

Energy efficiency and data transmission success rate of three algorithms is shown in Figure 4 and Figure 5. We can see that the node's energy efficiency and data transmission success rate reduce with its distance from the sink increasing when the node uses three different power control algorithm. As shown in Figure 4, MINPTC algorithm uses the minimum transmit power, the energy efficiency of MINPTC is the best among three algorithms. MAXPTC algorithm uses the maximum transmit power, the energy efficiency of MINPTC is the worst among three algorithms. The proposed algorithm can achieve better energy efficiency.

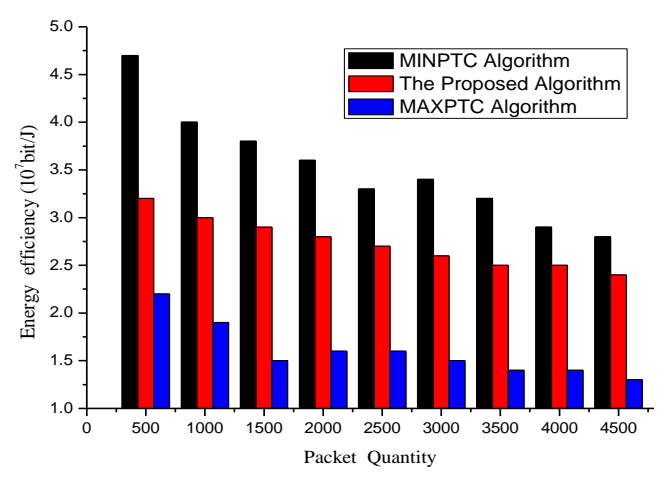

Figure 4. Energy Efficiency

As shown in Figure5, MINPTC algorithm uses the minimum transmit power, the energy efficiency of MINPTC is the best among three algorithms. But its transmission success rate is the worst. The reason is that the node uses minimum power can cause smaller SINR and interference ability, the average number of packets received less than the other two algorithms. Although the transmission success rate of MAXPTC is better its energy efficiency is the worst among three algorithms. The proposed algorithm can dynamically adjust the transmission power value to reach the Nash equilibrium which produces interference much lower than other two algorithms. So the proposed algorithm can achieve better energy efficiency and transmission success rate. 


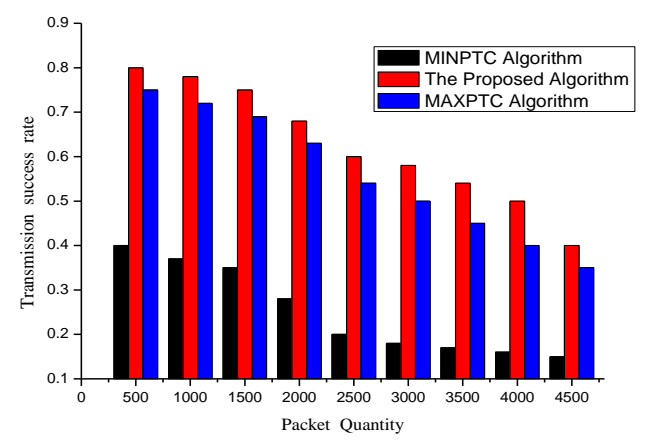

Figure 5. Data Transmission Success Rate

\section{Conclusion}

Power control in wireless sensor networks is an important issue due to the limited energy of the senor nodes. The power control protocol can help to decrease the transmission power of a node to a proper level and guarantee the link quality. In this paper, we presented a non-cooperative game algorithm with incomplete information to solve the power control problem in sensor networks. We apply Bayesian Nash equilibrium theorem to determine the existence and uniqueness proof of Nash equilibrium algorithm. Simulation results show that there exist points for each of the cost functions considered, which give the maximum net utility given the strategies taken by all other nodes as fixed. And the proposed algorithm is efficient and can achieve better performance.

\section{Acknowledgment}

This work is supported by Natural Science Foundation of Heilongjiang Province (F201104), Heilongjiang Province Education Department Foundation (12521416) and the Innovation Foundation of Harbin Bureau of Science and Technology (2011RFDXG005).

\section{References}

[1] W. S. Akyildiz, Y. Sankarasubramaniam and E. Cayirci, "A survey on sensor networks", Communications Magazine, IEEE, vol. 40, no. 8, (2002), pp. 102-114.

[2] F. Gunnarsson, "Power Control in Wireless Networks Characteristics and Fundamentals", Wireless Communications Systems and Networks, Kluwer Academic Press, (2004).

[3] J. Byres and G. Nasser, "Utility-based decision making in wireless sensor networks", Proceedings of the first ACM International Symposium on Mobile ad-hoc networking and Computing, (2000),pp. 143-144.

[4] S. Panichpapiboon and G. Ferrair, "O.K.Tonguz.Optimal transmit power in wireless sensor networks", IEEE Transactions on Mobile Computing, vol. 5, no. 10, (2006), pp. 1432-1447.

[5] M. Hayajneh and C. T. Abdallah, "Distributed joint rate and power control game-theoretic algorithms for wireless data", IEEE Communication Letter, vol. 8, no. 6, (2004), pp. 511-513.

[6] A. B. MacKenzie and S. B. Wicker, "Game theory in communications: Motivation, explanation, and application to power control", Proceedings to the IEEE Global Telecommunications Conference, San Antonio, TX, USA, (2001),pp. 821-826.

[7] M. Maskery and V. Krishnamurthy, "Decentralized adaptation in sensor networks: Analysis and application of regret-based algorithms", Proceedings of 46th IEEE Conference on Decision and Control, (2007), pp. 951956. 
[8] S. Cui, A. J. Goldsmith and A. Bahai, "Energy-efficiency of MIMO and cooperative MIMO techniques in sensor networks", IEEE Journal on Selected Areas in Communications, vol. 22, no. 6, (2004), pp. 1089-1098.

[9] S. Cui, A. J. Goldsmith and A. Bahai, "Energy constraint modulation optimization", IEEE Transactions on Wireless Communication, vol. 4, no. 5, (2005),pp. 2349-2360.

[10] Z. Wang, L. Jiang and C. He,"A Novel Price-Based Power Control Algorithm in Cognitive Radio Networks", IEEE Journals \& Magazines Communications Letters, vol. 17, no. 1, (2013),pp. 43-46.

[11] D. P. Dahnil and Y. P. Singh, "Adaptive clustering with transmission power control in wireless sensor networks", IET International Conference on Wireless Communications and Applications, (2012), pp. 1-6.

[12] R. Majumder, G. Bag and K. Ki-Hyung, "Power Sharing and Control in Distributed Generation With Wireless Sensor Networks", IEEE Transactions on Smart Grid, vol. 3, no. 2, (2012),pp. 618-634.

[13] L. Xu, D. T. Delaney, G. M. P. O'Hare and R. Collier, "The Impact of Transmission Power Control in Wireless Sensor Networks", IEEE International Symposium Network Computing and Applications, (2013), pp. 255-258.

[14] Y. Sun, S. Shan and C. Cui, "Power control strategy for wireless sensor networks based on node clustering", Wireless and Optical Communication Conference (WOCC), (2013), pp. 115-119.

[15] S. Haykin, "Cognitive radio: Brain-empowered wireless communications", IEEE Journal on Selected Areas inCommunications, vol. 23, no. 2, (2005), pp. 201-220.

[16] W. Jui Teng, "Admission Control With Distributed Joint Diversity and Power Control for Wireless Networks", IEEE Transactions on Vehicular Technology, vol. 58, no. 1, (2009), pp. 409-419.

[17] S. Sengupta, M. Chatterjee and K. A. Kwiat, "A Game Theoretic Framework for Power Control in Wireless Sensor Networks", IEEE Trans on Computers, vol. 59, no. 2, (2010), pp. 231-242.

[18] M. Meghji and D. Habibi, "Transmission power control in multihop wireless sensor networks", Proceedings of ICUFN, (2011), pp. 25-30.

[19] S. K. Jayaweera and T. Li, "Dynamic spectrum leasing in cognitive radio networks via primary-secondary user power control games”, IEEE Trans. Wir. Commun., vol. 8, no. 3, (2009), pp. 3300-3310.

[20] Y. Chen, "On cognitive radio networks with opportunistic power control strategies in fading channels", IEEE Trans. Wir. Commun., vol. 7, no. 1, (2008), pp. 2752-2761.

[21] W. Ren, Q. Zhao and A. Swami, "Power control in cognitive radio networks: How to cross a multi-lane highway”, IEEE J. Sel. Areas Commun., vol. 27, no. 5, (2009), pp. 1283-1296.

[22] Q. Jin, D. Yuan and Z. Guan, "Distributed geometric-programming based power control in cellular cognitive radio networks", Proc. of the 2009 IEEE 69th Vehic. Tech. Conf., Barcelona, Spain, (2009).

\section{Authors}

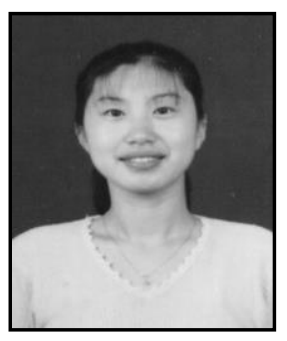

Guoyan Yang, She received her M.Sc. (2007) and Ph.D.(2012) in computer science and technology (2007) from Harbin Engineering University. Now she is full professor of College of Information and Technology, Heilongjiang University. Since 2012 he is rector of University. Her research interests include topology control, performance evaluation and routing algorithm in wireless sensor networks.

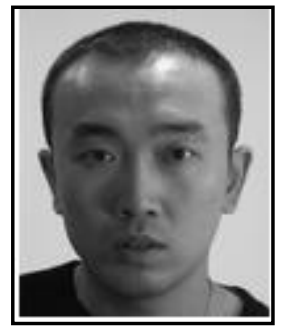

Xin Guan, He received the bachelor degree in School of Computer Science and Technology from Heilongjiang University, China in 2001 and the master degree from Harbin Institute of Technology, China in 2007. He received the Ph.D. at graduate school of science and technology, Keio University, Japan (2012). His research interests include topology control, performance evaluation and routing algorithm in wireless sensor networks. 
International Journal of Future Generation Communication and Networking Vol.7, No.1 (2014) 